Published in final edited form as:

N Engl J Med. 2013 July 11; 369(2): 145-154. doi:10.1056/NEJMoa1212914.

\title{
Cardiovascular Effects of Intensive Lifestyle Intervention in Type 2 Diabetes
}

\author{
The Look AHEAD Research Group
}

\section{Abstract}

BACKGROUND-Weight loss is recommended for overweight and obese individuals with type 2 diabetes based on short-term studies, but long-term effects on cardiovascular disease remain unknown. We examined whether intensive lifestyle intervention for weight loss decreased cardiovascular morbidity and mortality in overweight or obese adults with type 2 diabetes.

METHODS-We randomly assigned 5,145 overweight or obese individuals with type 2 diabetes recruited at 16 US centers to intensive lifestyle intervention (the intervention group), which promoted weight loss through decreased calorie intake and increased physical activity, or diabetes support and education (the control group). The primary outcome was the first post-randomization occurrence of a composite cardiovascular outcome (cardiovascular death, non-fatal myocardial infarction, non-fatal stroke, or hospitalized angina) over a planned maximum follow-up of 13.5 years.

RESULTS-The trial was stopped early based on a futility analysis when median follow-up was 9.6 years. Weight loss was greater in the intervention group than the control group throughout ( $8.6 \%$ vs. $0.7 \%$ at 1 year; $6.0 \%$ vs. $3.5 \%$ at study end). Intensive lifestyle intervention also produced greater reductions in hemoglobin A1c and greater initial improvements in fitness and all cardiovascular risk factors, except LDL cholesterol. The primary outcome occurred in 403 patients in the intervention group and in 418 in the control group (1.83/100 person-years and 1.92/100 person-years, respectively; hazard ratio 0.95 ; $95 \%$ CI 0.83 to $1.09, \mathrm{p}=0.505$ ).

CONCLUSION-In our study, intensive lifestyle intervention focused on weight loss did not reduce cardiovascular events in overweight or obese adults with type 2 diabetes. (Funded by the Department of Health and Human Services and others; ClinicalTrials.gov number,

NCT00017953.)

Weight loss is recommended for overweight and obese individuals with type 2 diabetes. ${ }^{1}$ This recommendation is based on short-term studies showing numerous benefits of weight loss, including improvements in glycemic control, cardiovascular disease risk factors, quality of life and other obesity-related comorbidities. ${ }^{2}$ However, it is unknown whether weight loss reduces the risk of cardiovascular morbidity and mortality in individuals with type 2 diabetes. Epidemiological studies in individuals with diabetes provide conflicting results, perhaps due to confounding from unintentional weight loss. ${ }^{3}$ A recent metaanalysis ${ }^{4}$ of cohort studies concluded that moderate intentional weight loss was associated with reduced mortality in "unhealthy" individuals, which included individuals with diabetes. The Swedish Obesity Subjects (SOS) study (ClinicalTrials.gov number NCT01479452) reported lower cardiovascular event rates over a mean follow-up of 13.3 years in patients with type 2 diabetes who underwent bariatric surgery; ${ }^{5}$ however, this was a non-randomized study, and the results achieved through surgery cannot be generalized to other approaches to weight loss.

Thus, a critical question remains: Does an intensive lifestyle intervention designed to produce weight loss through caloric restriction and increased physical activity decrease cardiovascular morbidity and mortality in overweight and obese adults with type 2 diabetes? 
Look AHEAD (Action for Health in Diabetes) addressed this question in a multicenter randomized clinical trial.

\section{METHODS}

\section{Study Design}

The study methods have been published previously. ${ }^{6,7}$ Look AHEAD was a randomized trial conducted at 16 clinical sites in the United States (see the Supplementary Appendix). The trial was designed and conducted by the authors, and all analyses were completed by the coordinating center. The study was approved by institutional review boards at each center. The trial was not blinded, but clinical assessors and end point adjudicators were masked to treatment assignment. The authors vouch for the accuracy and completeness of the data and all analyses and for the fidelity of this report to the trial protocol, which is available with the full text of this article at NEJM.org.

The study was sponsored by the National Institutes of Health, with additional support from other federal partners and the clinical research centers of several participating institutions. Major contributions were provided by the FedEx Corporation; Health Management Resources; LifeScan, Inc., a Johnson \& Johnson Company; Nestle HealthCare Nutrition, Inc.; Hoffmann-La Roche Inc.; Abbott Nutrition; and Unilever North America. None of the corporate sponsors had any role in the trial design, data analysis, or reporting of results.

\section{Participants}

To be eligible for participation in the trial, volunteers were required to be 45 to 76 years of age and to have all of the following: self-reported type 2 diabetes verified by use of glucoselowering medication, physician report, or glucose levels; body-mass index (BMI) of $25.0 \mathrm{~kg} /$ $\mathrm{m}^{2}$ or greater $\left(27 \mathrm{~kg} / \mathrm{m}^{2}\right.$ or greater in individuals taking insulin); hemoglobin A1c less than 11\%; systolic blood pressure less than $160 \mathrm{mmHg}$; diastolic blood pressure less than 100 $\mathrm{mmHg}$; triglycerides less than $600 \mathrm{mg} / \mathrm{dl}$; ability to complete a valid maximal exercise test suggesting it was safe to exercise; and a primary care provider. Participants could be using any type of glucose-lowering medication, but the percentage of participants using insulin was limited to less than $30 \%$. Participants with and without a history of cardiovascular disease were included to increase generalizeability of results. Additional eligibility criteria are described elsewhere ${ }^{6}$ and in the Supplementary Appendix.

\section{Interventions}

Eligible individuals were randomized to either an intensive lifestyle intervention (the intervention group) or diabetes support and education (the control group); randomization was stratified by clinical site. Curricula for both treatment arms were developed centrally and have been described in detail ${ }^{6,8}$ (see the Supplementary Appendix). Intensive lifestyle intervention aimed at achieving and maintaining at least a 7\% weight loss by focusing on reduced caloric intake and increased physical activity. The program included both group and individual counseling sessions, occurring weekly during the first six months, with decreasing frequency over the course of the trial. Specific intervention strategies included a calorie goal of 1200 to $1800 \mathrm{kcal} / \mathrm{day}$ (with less than $30 \%$ of calories from fat and more than $15 \%$ from protein), use of meal replacement products, and at least 175 minutes per week of moderate intensity physical activity. A toolbox of strategies was available for participants having difficulty achieving the weight loss goals.

Diabetes support and education featured three group sessions per year focused on diet, exercise, and social support during years 1 to 4 . In subsequent years the frequency was reduced to one session annually. 
All medication adjustments were made by the participant's health care provider, with the exception of temporary changes in glucose-lowering medications made by study staff to reduce the risk of hypoglycemia in the intensive lifestyle intervention group. Participants and their health care providers received annual reports on the participants' updated cardiovascular risk factors and the goals recommended by the American Diabetes Association. ${ }^{1}$

\section{Assessments and Outcomes}

Certified staff, masked to participants' intervention assignment, measured weight, waist circumference and blood pressure, assessed medication use, and obtained blood for analysis at the central laboratory annually. ${ }^{6}$ Maximal exercise tests were performed on the full cohort prior to randomization. Submaximal exercise tests were performed on the full cohort at years 1 and 4 and on a subset at year 2 .

During annual visits and 6-month phone calls, participants were queried by staff masked to treatment assignment about all medical events and hospitalizations. These queries were augmented with searches of national databases on deaths. Hospital and other records were obtained for potential cardiovascular events and adjudicated according to standard criteria by reviewers masked to intervention assignment (see Supplementary Appendix).

The primary end point was the first post-randomization occurrence of a composite cardiovascular outcome. Initially, the composite cardiovascular outcome included cardiovascular death, nonfatal myocardial infarction, and nonfatal stroke, and the anticipated maximal follow-up interval was 11.5 years. During the first two years of the trial, the primary event rate in the control group was lower than expected. ${ }^{9}$ Therefore, hospitalized angina was added to the primary outcome and planned follow-up was extended to a maximum of 13.5 years. Three composite secondary cardiovascular outcomes were also examined: cardiovascular death, myocardial infarction, or stroke (the original primary outcome); death (all causes), myocardial infarction, stroke, or hospitalized angina; and death (all causes), myocardial infarction, stroke, hospitalized angina, coronary artery bypass grafting, percutaneous coronary angioplasty, hospitalization for heart failure, or peripheral vascular disease.

\section{Statistical Analyses}

With a planned sample size of 5000 participants, Look AHEAD had greater than $80 \%$ probability of detecting an $18 \%$ difference in major cardiovascular events between the two groups, assuming a two-sided alpha level of 0.05 , a primary outcome rate of $2 \%$ per year in the control group, and a planned maximum follow-up of 13.5 years. The $18 \%$ difference was chosen based on reductions in mortality in individuals with type 2 diabetes with voluntary weight loss in an observational study, ${ }^{10}$ effect sizes chosen for trials with similar outcomes, ${ }^{11}$ feasibility, and public health significance.

On September 14, 2012, Look AHEAD's primary sponsors, based on futility analysis and recommendation of the data and safety monitoring board, instructed the study investigators to terminate the intervention. All data analyses extend to this date. At that time, the probability of observing a significant positive result at planned end of follow-up, assuming the actual hazard ratio to be 0.82 , was estimated to be $1 \%$.

Baseline characteristics and key safety outcomes were compared between study groups using chi-square, Fisher's exact, Wilcoxon rank-sum, two-sample t-tests and Poisson regression. Physical and laboratory measurements and medication use from baseline through 10 years were modeled with generalized linear regression and generalized estimating equations. Center was included as a covariate, covariance was unstructured, and linear 
contrasts were used to compare groups throughout post-baseline follow-up and at annual visits. Analyses of primary and secondary outcomes were performed using time-to-event methods according to the intention-to-treat principle as pre-specified in the protocol. Kaplan-Meier estimates were used to calculate the proportion of participants who had an event over time. First occurrences of primary and secondary outcomes in the two groups were compared with hazard ratios and $95 \%$ confidence intervals. Two-sided p-values were calculated with likelihood-ratio tests from Cox proportional-hazards regression, with models containing terms for clinical site, history of cardiovascular disease, and intervention assignment. The consistency of intervention effects on the primary outcome among three pre-specified subgroups (sex, race or ethnicity and history of cardiovascular disease) was evaluated using interaction tests. Results were not adjusted for multiple comparisons and $\mathrm{p}<0.05$ was used. Statistical analyses were conducted using S-Plus software, version 8.0 (Insightful) or SAS software, and version 9.1 (SAS Institute).

\section{RESULTS}

\section{Study Participants}

Between August 2001 and April 2004, a total of 5145 participants were enrolled and randomly assigned to intensive lifestyle intervention $(\mathrm{N}=2,570)$ or to diabetes support and education $(\mathrm{N}=2,575)$ (Supplementary Appendix Fig. S1). Groups were similar at baseline (Table 1). The average age was 58.7 years, $59 \%$ were women, and the mean BMI was 36.0 $\mathrm{kg} / \mathrm{m}^{2}$. The median duration of diabetes was 5 years, and $14 \%$ reported a history of cardiovascular disease. Additional baseline data have been published previously. ${ }^{12}$

When the intervention was stopped on September 14, 2012, the median follow-up was 9.6 years (interquartile range 8.9 to 10.3 ) and fewer than $4 \%$ of all randomized participants had been lost to follow-up.

\section{Weight, Waist Circumference and Fitness}

Intensive lifestyle intervention produced significantly greater changes than diabetes support and education in weight, waist circumference and fitness (Fig. 1A-C) and Supplementary Appendix Table S1). Differences in mean weight loss were greatest at 1 year $(8.6 \%$ in the intervention group vs. $0.7 \%$ in the control group) but remained statistically significant throughout the trial. When intervention ended, mean weight loss from baseline was $6.0 \%$ in the intervention group compared with $3.5 \%$ in the control group.

\section{Cardiovascular Disease Risk Factors}

During the first year of follow-up, intensive lifestyle intervention produced greater improvements than diabetes support and education in all measured cardiovascular risk factors except low-density lipoprotein (LDL) cholesterol (Fig. 1D and Supplementary Appendix Fig. S2 and Table S1). The difference in cardiovascular risk factors between intervention groups diminished over time, with hemoglobin A1c and systolic blood pressure showing the most sustained differences. LDL cholesterol levels were lower in the control group than the intervention group (mean difference of $1.6 \mathrm{mg} / \mathrm{dl}$ over the 10 years). Use of antihypertensive medications, statins and insulin was lower in the intervention group than in the control group (Supplementary Appendix Fig. S2 and Fig. S3 and Table S1).

\section{Clinical Outcomes}

The composite primary outcome of the first occurrence of hospitalized angina, nonfatal myocardial infarction, nonfatal stroke, or death from cardiovascular causes occurred in 821 participants (403 in the intensive lifestyle intervention group and 418 in the diabetes support and education group). Rates were not different between groups (1.83/100 person-years and 
$1.92 / 100$ person-years, respectively; hazard ratio $=0.95$; $95 \%$ CI 0.83 to $1.09, \mathrm{p}=0.505$ )

(Table 2 and Fig. 2). There were also no significant differences between the two trial groups with respect to the pre-specified composite secondary outcomes or any of the individual cardiovascular events making up the composite outcomes (Table 2). There were no significant interactions among the pre-specified subgroups (Fig. 3).

\section{Adverse Events}

Severe hypoglycemia, gallstones, fractures, amputations and congestive heart failure were monitored, as plausibly being affected by intensive lifestyle intervention. Although the rate of self-reported fractures differed significantly between groups (2.16/100 person-years in the control group versus $2.51 / 100$ person-years in the intervention group; $\mathrm{p}=0.011$ ), the incidence rate for adjudicated fractures did not (1.64/100 person-years and 1.66/100 personyears, respectively; $\mathrm{p}=0.833$ ) (Supplementary Appendix Table S2).

\section{DISCUSSION}

The Look AHEAD trial compared the effect of an intensive lifestyle intervention with a control regimen of diabetes support and education in overweight and obese participants with type 2 diabetes. At a median follow-up of almost 10 years, there was no significant difference between the two groups in cardiovascular morbidity and mortality.

Look AHEAD showed that overweight and obese adults with type 2 diabetes can lose weight and maintain modest weight losses over 10 years. Multi-component lifestyle interventions in clinical trial settings typically produce initial weight losses of 7 to $10 \% ; 13,14$ with maximal weight loss at 1 year, followed by gradual regain. ${ }^{13-15}$ However, few studies have provided ongoing intervention for an extended time. ${ }^{16}$ In our trial, the initial mean weight loss in the intensive lifestyle intervention group was $8.6 \%$. This was followed by weight regain through year 5 and then a subsequent gradual decrease in weight, resulting in an average weight loss of $6.0 \%$ at the end of the trial. The control group experienced a gradual but consistent weight loss throughout the study, resulting in an average weight loss of $3.5 \%$ at end of the trial. The intervention group also had greater improvements in fitness, particularly at 1 year.

We have considered several possible explanations for the lack of a significant difference in the cardiovascular event rates between groups. One possibility is that the study lacked sufficient power. We do not feel this explains the negative result; the $95 \%$ confidence interval for the primary outcome excluded the benefit of $18 \%$ or more targeted in the trial's design. Another possibility is that sustained weight losses larger than those achieved in Look AHEAD may be required to reduce the risk of cardiovascular disease. In this regard, it is noteworthy that the differential weight loss between the two trial groups averaged $4 \%$ over the course of the study but only $2.5 \%$ at the end. However, our trial was planned to test the effects of intensive lifestyle intervention and the weight losses achieved in the intervention group are representative of the best that has been achieved with current lifestyle approaches. Third, educational sessions provided to the control group assigned to diabetes support and education, the greater use of statinsin the control group, and the overall intensification of medical management of cardiovascular risk factors ${ }^{17}$ may have made a relative benefit of intensive lifestyle intervention more difficult to demonstrate. The intervention may also have had different effects in different subgroups. Although none of the interactions with subgroups were statistically significant, our data suggest that the event rate for the primary outcome was non-significantly lower in ILI than in DSE among individuals with no history of CVD at baseline, but non-significantly higher in ILI than in DSE among individuals with CVD at baseline. 
There are several limitations to these findings. We used a specific lifestyle intervention that focused on achieving weight loss through caloric restriction and increased physical activity. It is unclear whether an intervention focused on changes in dietary composition, for example the Mediterranean diet, ${ }^{18}$ might yield different outcomes. In addition, Look AHEAD recruited participants with type 2 diabetes who were motivated to lose weight through lifestyle intervention and who could successfully complete a maximal fitness test at baseline. Thus, the results cannot be generalized to all individuals with type 2 diabetes.

The finding that intensive lifestyle intervention, compared with diabetes support and education, did not reduce the risk of cardiovascular morbidity and mortality must be considered within the context of other positive effects observed with this intervention. Intensive lifestyle intervention produced clinically meaningful improvements in hemoglobin A1c, especially over the first year of the intervention, that were at least partly sustained throughout follow-up. This positive effect is noteworthy since it may explain why participants in the intervention group were less likely to be treated with insulin over this period. Further, we recently reported that individuals in the intervention group of Look AHEAD were more likely to experience partial diabetes remission during the first four years of the trial. ${ }^{19}$ Other benefits identified during the early years of the trial have included improvements in urinary incontinence, ${ }^{20}$ sleep apnea, ${ }^{21}$ depression, ${ }^{22}$ quality of life, ${ }^{23}$ physical function, ${ }^{24}$ and mobility. Intensive lifestyle intervention has also been shown to prevent or delay the development of type 2 diabetes in other studies. ${ }^{15,26}$

In conclusion, the Look AHEAD trial found that intensive lifestyle intervention did not reduce the risk of cardiovascular morbidity or mortality compared with a control program of diabetes support and education in overweight and obese participants with type 2 diabetes.

\section{Supplementary Material}

Refer to Web version on PubMed Central for supplementary material.

\section{Acknowledgments}

The Look AHEAD Research Group acknowledges the contributions of Drs. Frederick Brancati and Richard Rubin, who passed away during the completion of this manuscript. Both colleagues were fundamental to the successful development and execution of our trial.

\section{Appendix}

$\underline{\text { Authors }}$

Rena R.Wing, Ph.D.; Paula Bolin, R.N., M.C.; Frederick L.Brancati, M.D., M.H.S.\#; George A.Bray, M.D.; Jeanne M.Clark, M.D., M.P.H.; MaceCoday, Ph.D.; Richard S.Crow, M.D\#; Jeffrey M.Curtis, M.D., M.P.H.; CaitlinEgan, M.S.; Mark A.Espeland, Ph.D.; MaryEvans, Ph.D.; John P.Foreyt, Ph.D.; SiranGhazarian, M.D.; Edward W.Gregg, Ph.D.; BarbaraHarrison, M.S.; Helen P.Hazuda, Ph.D.; James O.Hill, Ph.D.; Edward S.Horton, M.D.; Van S.Hubbard, M.D., Ph.D.; John M.Jakicic, Ph.D.; Robert W.Jeffery, Ph.D.; Karen C.Johnson, M.D., M.P.H.; Steven E.Kahn, M.B., Ch.B.; Abbas E.Kitabchi, Ph.D., M.D.; William C.Knowler, M.D., Dr.P.H.; Cora E.Lewis, M.D., M.S.P.H.; Barbara J.MaschakCarey, M.S.N., C.D.E.; Maria G.Montez, R.N., M.S.H.P., C.D.E.; AnneMurillo, B.S.; David M.Nathan, M.D.; JenniferPatricio, M.S.; AnnePeters, M.D.; XavierPi-Sunyer, M.D.; HenryPownall, Ph.D.; DavidReboussin, Ph.D.; JudithRegensteiner, Ph.D.; Amy D.Rickman, Ph.D., R.D., L.D.N.; Donna H.Ryan, M.D.; MonikaSafford, M.D.; Thomas A.Wadden, Ph.D.; Lynne E.Wagenknecht, Dr.P.H.; Delia S.West, Ph.D.; David F.Williamson, Ph.D.; Susan Z.Yanovski, M.D. 


\section{Look AHEAD Funding and Support}

This study is supported by the Department of Health and Human Services through the following cooperative agreements from the National Institutes of Health: DK57136, DK57149, DK56990, DK57177, DK57171, DK57151, DK57182, DK57131, DK57002, DK57078, DK57154, DK57178, DK57219, DK57008, DK57135, and DK56992. The following federal agencies have contributed support: National Institute of Diabetes and Digestive and Kidney Diseases; National Heart, Lung, and Blood Institute; National Institute of Nursing Research; National Center on Minority Health and Health Disparities; NIH Office of Research on Women's Health; and the Centers for Disease Control and Prevention. This research was supported in part by the Intramural Research Program of the National Institute of Diabetes and Digestive and Kidney Diseases. The Indian Health Service (I.H.S.) provided personnel, medical oversight, and use of facilities. The opinions expressed in this paper are those of the authors and do not necessarily reflect the views of the I.H.S. or other funding sources.

Additional support was received from The Johns Hopkins Medical Institutions Bayview General Clinical Research Center (M01RR02719); the Massachusetts General Hospital Mallinckrodt General Clinical Research Center and the Massachusetts Institute of Technology General Clinical Research Center (M01RR01066); the University of Colorado Health Sciences Center General Clinical Research Center (M01RR00051) and Clinical Nutrition Research Unit (P30 DK48520); the University of Tennessee at Memphis General Clinical Research Center (M01RR0021140); the University of Pittsburgh General Clinical Research Center (GCRC) (M01RR000056), the Clinical Translational Research Center (CTRC) funded by the Clinical \& Translational Science Award (UL1 RR 024153) and NIH grant (DK 046204); the VA Puget Sound Health Care System Medical Research Service, Department of Veterans Affairs; and the Frederic C. Bartter General Clinical Research Center (M01RR01346).

The following organizations have committed to make major contributions to Look AHEAD: FedEx Corporation; Health Management Resources; LifeScan, Inc., a Johnson \& Johnson Company; OPTIFAST® of Nestle HealthCare Nutrition, Inc.; Hoffmann-La Roche Inc.; Abbott Nutrition; and Slim-Fast Brand of Unilever North America.

Some of the information contained herein was derived from data provided by the Bureau of Vital Statistics, New York City Department of Health and Mental Hygiene.

\section{REFERENCES}

1. American Diabetes Association. Clinical Practice Recommendations. American Diabetes Association; Alexandria, VA: 2013.

2. NHLBI. Clinical guidelines on the identification, evaluation, and treatment of overweight and obesity in adults-The evidence report. Obes Res. 1998; 6(S2):51S-210S. [PubMed: 9813653]

3. Williamson DF. Weight loss and mortality in persons with type-2 diabetes mellitus: A review of the epidemiological evidence. Exp Clin Endocrinol Diabetes. 1998; 106(Suppl 2):14-21. [PubMed: 9792476]

4. Harrington M, Gibson S, Cottrell RC. A review and meta-analysis of the effect of weight loss on allcause mortality risk. Nurt Res Rev. 2009; 22(1):93-108.

5. Romeo S, Maglio C, Burza MA, et al. Cardiovascular events after bariatric surgery in obese subjects with type 2 diabetes. Diabetes Care. 2012; 35(12):2613-7. [PubMed: 22855732]

6. Look AHEAD Research Group. Look AHEAD: Action for Health in Diabetes: Design and methods for a clinical trial of weight loss for the prevention of cardiovascular disease in type 2 diabetes. Control Clin Trials. 2003; 24:610-28. [PubMed: 14500058]

7. Look AHEAD Action for Health in Diabetes. Accessed at https://www.lookaheadtrial.org 
8. Look AHEAD Research Group. The Look AHEAD study: A description of the lifestyle intervention and the evidence supporting it. Obesity (Silver Spring). 2006; 14(5):737-52. [PubMed: 16855180]

9. Brancati FL, Evans M, Furberg CD, et al. Midcourse correction to a clinical trial when the event rate is underestimated: the Look AHEAD (Action for Health in Diabetes) Study. Clin Trials. 2012; 9(1): 113-24. [PubMed: 22334468]

10. Williamson DF, Thompson TJ, Thun M, Flanders D, Pamuk E, Byers T. Intentional weight loss and mortality among overweight individuals with diabetes. Diabetes care. 2000; 23(10):1499-504. [PubMed: 11023143]

11. Buse JB, Bigger JT, Byington RP, et al. Action to Control Cardiovascular Risk in Diabetes (ACCORD) trial: design and methods. Am J Cardiol. 2007; 99(12A):21i-33i.

12. Bray G, Gregg E, Haffner S, et al. Baseline characteristics of the randomized cohort from the Look AHEAD (Action for Health in Diabetes) study. Diab Vasc Dis Res. 2006; 3(3):202-15. [PubMed: 17160917]

13. Wing, RR. Behavioral approaches to the treatment of obesity. In: Bray, G.; Bouchard, C., editors. Handbook of Obesity: Clinical Applications. 2nd ed.. Marcel Dekker, Inc.; New York: 2004.

14. Wadden TA, Webb VL, Moran CH, Bailer BA. Lifestyle modification for obesity: new developments in diet, physical activity, and behavior therapy. Circulation. 125(9):1157-70. [PubMed: 22392863]

15. Knowler WC, Barrett-Connor E, Fowler SE, et al. Reduction in the incidence of type 2 diabetes with lifestyle intervention or metformin. N Engl J Med. 2002; 346(6):393-403. [PubMed: 11832527]

16. Knowler WC, Fowler SE, Hamman RF, et al. 10-year follow-up of diabetes incidence and weight loss in the Diabetes Prevention Program Outcomes Study. Lancet. 2009; 374(9702):1677-86. [PubMed: 19878986]

17. Ali MK, Bullard KM, Saaddine JB, Cowie CC, Imperatore G, Gregg EW. Achievement of goals in U.S. diabetes care, 1999-2010. N Engl J Med. 2013; 368(17):1613-24. [PubMed: 23614587]

18. Estruch R, Ros E, Salas-Salvado J, et al. Primary prevention of cardiovascular disease with a Mediterranean diet. N Engl J Med. 2013

19. Gregg EW, Chen H, Wagenknecht LE, et al. Association of an intensive lifestyle intervention with remission of type 2 diabetes. JAMA. 2012; 308(23):2489-96. [PubMed: 23288372]

20. Phelan S, Kanaya AM, Subak LL, et al. Weight loss prevents urinary incontinence in women with type 2 diabetes: results from the Look AHEAD trial. J Urol. 2012; 187(3):939-44. [PubMed: 22264468]

21. Foster GD, Borradaile KE, Sanders MH, Millman R, Zammit G, Newman AB, Wadden TA, Kelley D, Wing RR, Pi-Sunyer FX, Reboussin D, Kuna ST. A randomized study on the effects of weight loss on obstructive sleep apnea among obese patients with type 2 diabetes: the Sleep AHEAD study. Arch Intern Med. 2009; 169(17):1619-26. [PubMed: 19786682]

22. Faulconbridge LF, Wadden TA, Rubin RR, et al. One-year changes in symptoms of depression and weight in overweight/obese individuals with type 2 diabetes in the Look AHEAD study. Obesity (Silver Spring). 2012; 20(4):783-93. [PubMed: 22016099]

23. Williamson DA, Rejeski J, Lang W, Van Dorsten B, Fabricatore AN, Toledo K. Impact of a weight management program on health-related quality of life in overweight adults with type 2 diabetes. Arch Intern Med. 2009; 169(2):163-71. [PubMed: 19171813]

24. Foy CG, Lewis CE, Hairston KG, et al. Intensive lifestyle intervention improves physical function among obese adults with knee pain: findings from the Look AHEAD trial. Obesity (Silver Spring). 2011; 19(1):83-93. [PubMed: 20559303]

25. Rejeski WJ, Ip EH, Bertoni AG, et al. Lifestyle change and mobility in obese adults with type 2 diabetes. N Engl J Med. 2012; 366(13):1209-17. [PubMed: 22455415]

26. Tuomilehto J, Lindstrom J, Eriksson J, et al. Prevention of type 2 diabetes mellitus by changes in lifestyle among subjects with impaired glucose tolerence. N Engl J Med. 2001; 344:1343-50.

[PubMed: 11333990] 

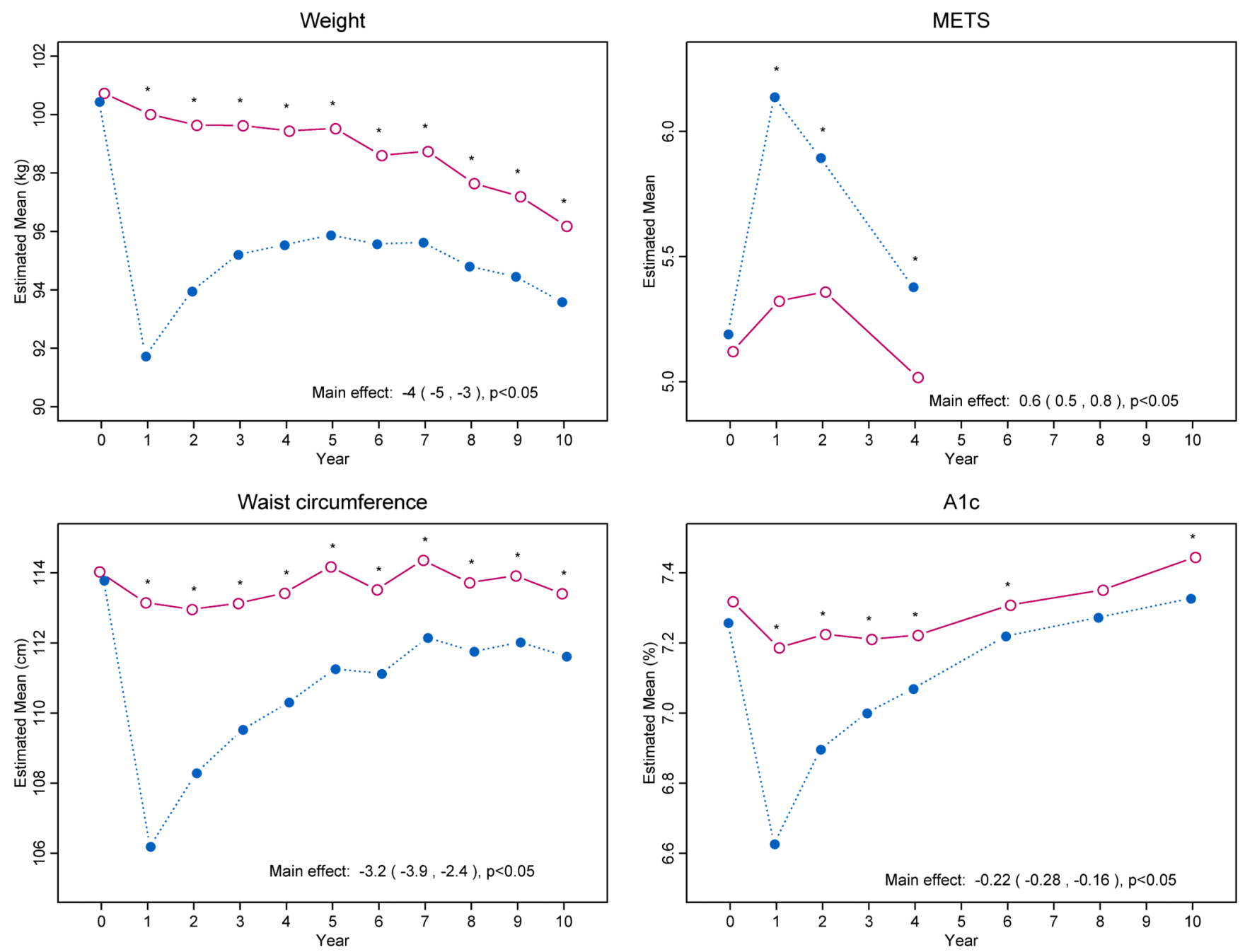

Figure 1.

Changes in Weight, Physical Fitness, Waist Circumference, and Glycated Hemoglobin Levels during 10 Years of Follow-up. 


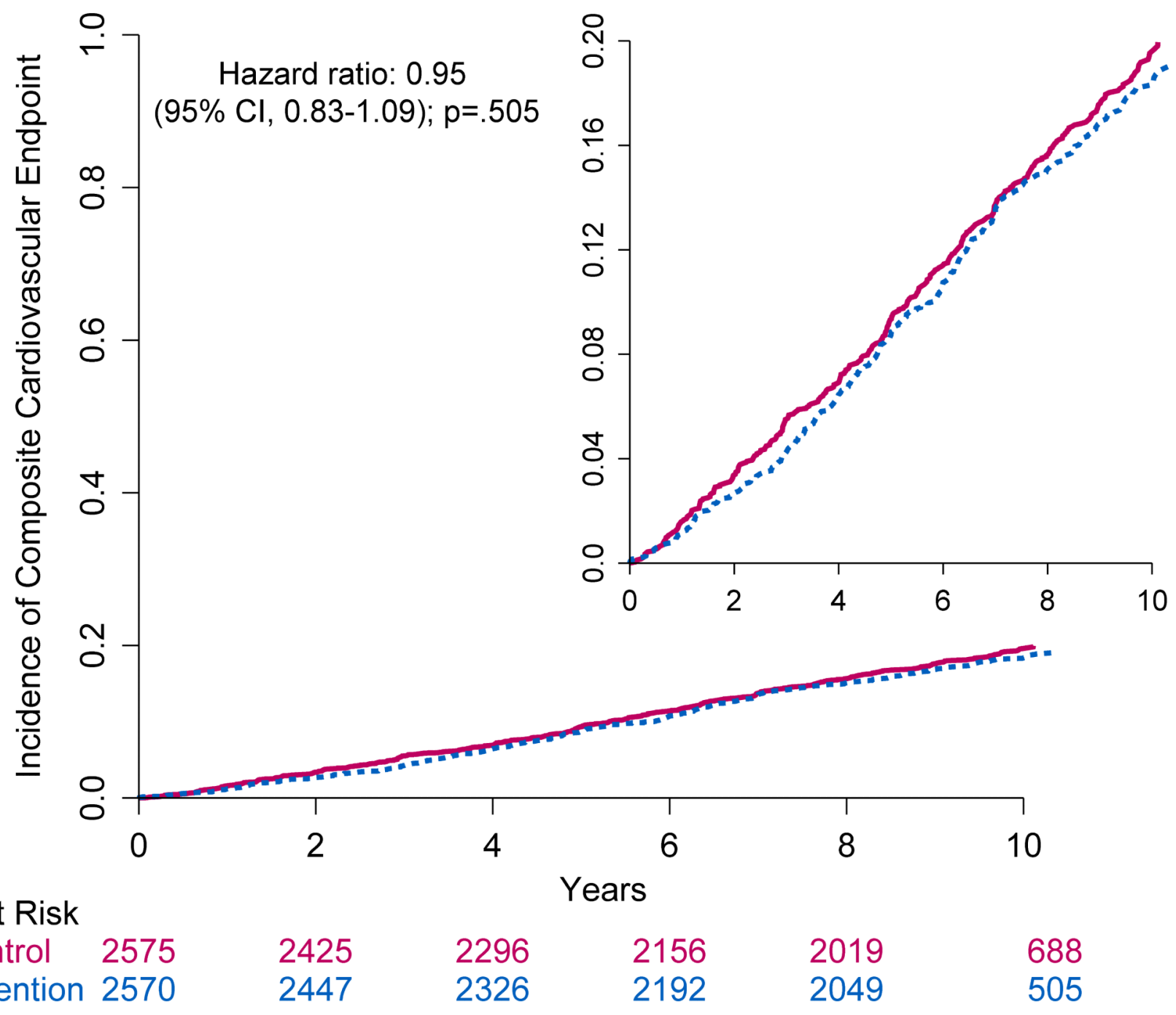

Figure 2.

Cumulative Hazard Curves for the Primary Composite End Point. 
Page 11

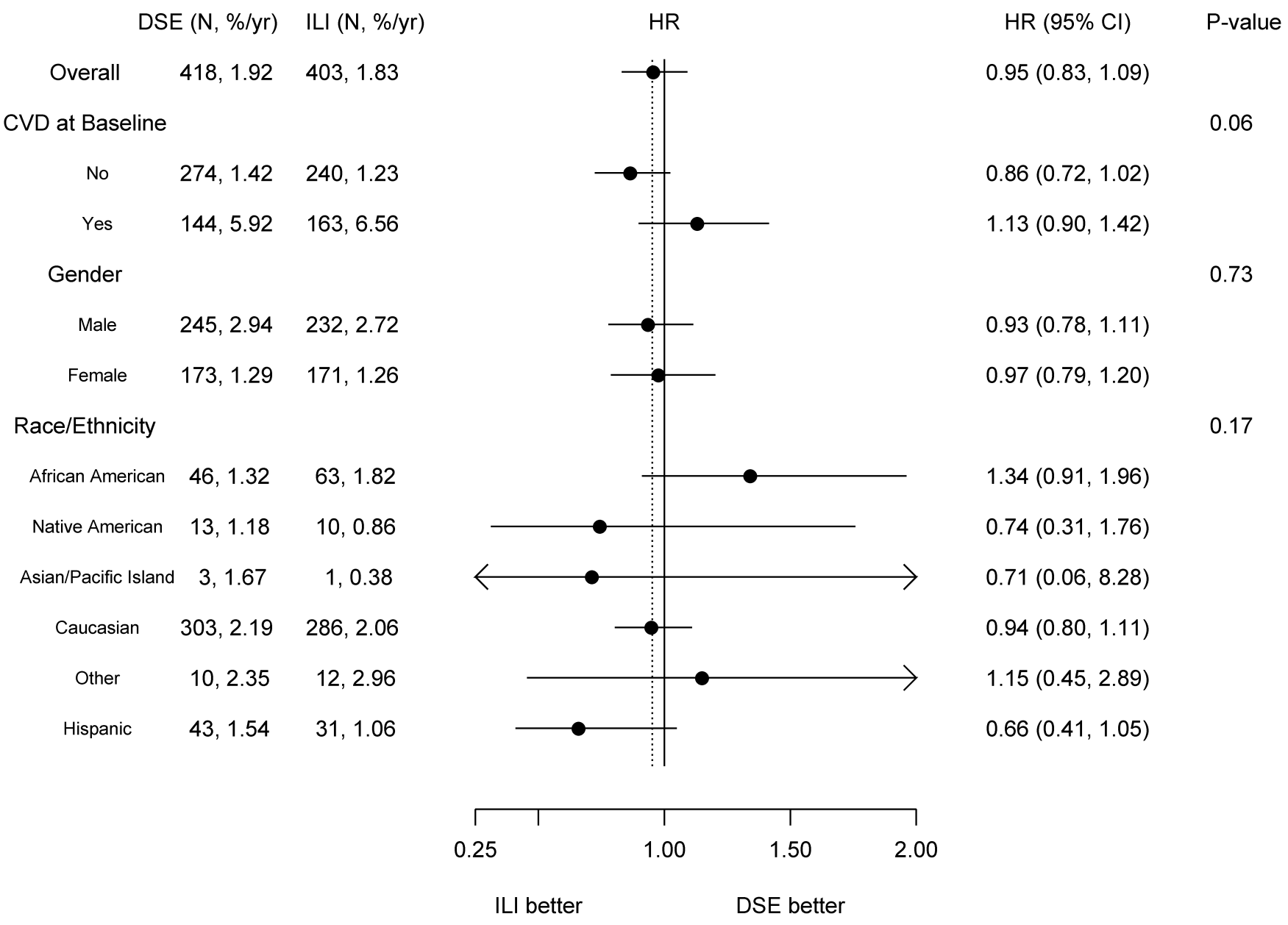

Figure 3.

Primary Outcome in Prespecified Subgroups. 
Table 1

Comparison of intervention groups at baseline.

\begin{tabular}{|c|c|c|c|}
\hline Variable & & DSE & ILI \\
\hline Age & Mean (SD) & $58.9(6.9)$ & $58.6(6.8)$ \\
\hline Female & $\mathrm{N}(\%)$ & $1537(59.7)$ & $1526(59.4)$ \\
\hline \multirow[t]{7}{*}{ Race/ethnicity } & $\mathrm{N}(\%)$ & & \\
\hline & African American & $404(15.7)$ & $400(15.6)$ \\
\hline & American Indian & $128(5.0)$ & $130(5.1)$ \\
\hline & Asian/Pacific Islander & $21(0.8)$ & $29(1.1)$ \\
\hline & White & $1631(63.3)$ & $1621(63.1)$ \\
\hline & Hispanic & $340(13.2)$ & $340(13.2)$ \\
\hline & Other/Mixed & $51(2.0)$ & $49(1.9)$ \\
\hline History of CVD ${ }^{*}$ & $\mathrm{~N}(\%)$ & $348(13.5)$ & $366(14.2)$ \\
\hline Using insulin & $\mathrm{N}(\%)$ & $410(16.5)$ & $382(15.4)$ \\
\hline Smoking & $\mathrm{N}(\%)$ & $110(4)$ & $117(5)$ \\
\hline $\begin{array}{c}\text { Duration of diabetes } \\
\text { (years) }\end{array}$ & Median (Q1,Q3) & $5.0(2.0,10)$ & $5.0(2.0,10)$ \\
\hline Weight $(\mathrm{kg})$ & Mean (SD) & 101 ( 19) & $101(20)$ \\
\hline BMI $\left(\mathrm{kg} / \mathrm{m}^{2}\right)$ & Mean (SD) & $36.0(5.8)$ & $35.9(6.0)$ \\
\hline Waist circumference $(\mathrm{cm})$ & Mean (SD) & $114(14)$ & $114(14)$ \\
\hline A1c $(\%)$ & Mean (SD) & $7.3(1.2)$ & $7.2(1.1)$ \\
\hline $\mathrm{SBP}(\mathrm{mm} \mathrm{Hg})^{* *}$ & Mean (SD) & $129(17)$ & $128(17)$ \\
\hline $\mathrm{DBP}(\mathrm{mm} \mathrm{Hg})$ & Mean (SD) & $70.4(9.6)$ & $69.9(9.5)$ \\
\hline HDL-C (mg/dl) & Mean (SD) & $43.5(12)$ & $43.4(12)$ \\
\hline LDL-C (mg/dl) & Mean (SD) & $112(32)$ & $112(32)$ \\
\hline Triglycerides $(\mathrm{mg} / \mathrm{dl})$ & Median (Q1,Q3) & $152(107,218)$ & $155(110,221)$ \\
\hline
\end{tabular}

$\mathrm{ILI}=$ Intensive Lifestyle Intervention. DSE = Diabetes Support and Education.

Prior CVD was defined as history of myocardial infarction, stroke, congestive heart failure or CVD procedures (CABG, PTCA, carotid endarterectomy, angioplasty of a lower extremity artery, or aortic aneurysm repair).

** indicates significant difference between arms with $\mathrm{p}<0.01$. 
